\title{
TIPIFICACIÓN COROLÓGICA DE LOS MICROMAMÍFEROS IBÉRICOS EN RELACIÓN CON EUROPA Y ÁFRICA
}

\author{
R. Real ${ }^{1}$, J. C. Guerrero, A. L. Márquez, J. Olivero y J. M. Vargas
}

\begin{abstract}
RESUMEN
En el presente artículo se analizan los diferentes tipos de distribución, o corotipos, que pudieran existir para las 70 especies de micromamíferos que se distribuyen por los 12 sistemas montañosos que circundan la parte más occidental de la cuenca mediterránea. También se ha llevado a cabo un análisis de las tendencias de la riqueza de especies en relación con los principales gradientes ambientales. Se han identificado cinco corotipos que incluyen la distribución de 49 especies: un corotipo ampliamente distribuido (I), dos corotipos ibéricos (II y III), un corotipo alpino (IV) y un corotipo africano (V). Únicamente los corotipos II, IV y V se han podido caracterizar ambientalmente. El corotipo II se corresponde con aquellos sistemas montañosos que presentan una disponibilidad intermedia de agua procedente de la lluvia. El corotipo IV incluye el sistema montañoso con mayor precipitación e inestabilidad climática. El corotipo V se caracteriza por abarcar los sistemas montañosos con una mayor disponibilidad de energía. Para el conjunto de los micromamíferos y los no roedores el número de especies presenta una tendencia bimodal respecto a la disponibilidad de energía. Para los roedores es la disponibilidad simultánea de agua y energía el principal factor que explica la variación de riqueza de especies, también de forma bimodal.
\end{abstract}

Palabras clave: corología, riqueza específica, Erinaceomorpha, Soricomorpha, Rodentia, corotipos, sistemas montañosos, Europa Occidental, Norte de África, Península Ibérica.

\section{ABSTRACT \\ Chorological typification of iberian micromammals in relation to Europe and Africa}

In this paper we analysed the different types of distribution, or chorotypes, existing for the 70 species of micro-mammals distributed by the 12 mountain ranges surrounding the westernmost part of the Mediterranean basin. We also analysed the species richness' trends in relation to the main environmental gradients. We identified five chorotypes involving the distributions of 49 species: a widespread chorotype (I), two Iberian chorotypes (II and III), an Alpine chorotype (IV), and an African chorotype (V). Only chorotypes II, IV, and V were environmentally characterised. Chorotype II corresponds to the mountain ranges with intermediate precipitation. Chorotype IV includes the range with the highest precipitation and climatic instability. Chorotype V involves the mountain ranges with highest energy availability. For non-rodents and for all micro-mammals together, the number of species shows a bimodal trend with respect to energy availability. For rodents this bimodal trend occurs mainly with respect to the simultaneous availability of water and energy.

Keywords: chorology, species richness, Erinaceomorpha, Soricomorpha, Rodentia, chorotypes, mountain ranges, Western Europe, North Africa, Iberian Peninsula.

Departamento de Biología Animal, Facultad de Ciencias, Universidad de Málaga, 29071 Málaga, España.

rrgimenez@uma.es 


\section{Introducción}

El análisis de la fauna de micromamíferos en las montañas del Paleártico occidental ha dado lugar al reconocimiento de una clara diferenciación entre la composición de especies europea y la composición africana (Vargas et al., 2003), afirmación que es válida tanto para los micromamíferos roedores como para los no roedores (erinaceomorfos y soricomorfos). Dentro del contexto europeo, según la misma fuente, tan sólo aparece una frontera biótica débil para este último grupo de especies. Como consecuencia de ello, los Pirineos se unen al Macizo Central Francés y a los Alpes en una misma región biótica, mientras que los sistemas montañosos exclusivamente ibéricos configuran, por sí solos, otra región distinta. Esta situación sugiere diferentes posibilidades en cuanto a los orígenes de la fauna de micromamíferos de la Península Ibérica: un origen endémico; un origen europeo, dado el papel de refugio cuaternario desempeñado por la Península Ibérica para ciertos grupos de fauna eurosiberiana (de Lattin, 1967); y un origen africano, favorecido por la afinidad ecológica entre Iberia y el continente meridional, si bien dificultado por la importante barrera que representa el brazo de mar del Estrecho de Gibraltar.

El patrón de regionalización descrito en Vargas et al. (2003) para los micromamíferos se complementa con un análisis de los procesos de intercambio de especies entre sistemas montañosos, bajo la perspectiva de una historia biogeográfica dominada por la dispersión. En dicho análisis se vislumbra la existencia de dos núcleos geográficos de intercambio principal de especies: la Europa transpirenaica, cuya relación faunística con la Península Ibérica resulta más evidente, y el norte de África.

El objetivo del presente trabajo es profundizar en el estudio de la fauna de micromamíferos del Paleártico Occidental, con el fin de identificar biotas diferentes en dicha zona y arrojar luz acerca del modo en que éstas han influido sobre la fauna de micromamíferos ibéricos. El estudio se aborda desde dos enfoques distintos: 1) la definición de los tipos de distribución principales que presentan los micromamíferos en Iberia y su entorno geográfico; y 2) el análisis de los patrones de riqueza específica del grupo en cuestión y la forma en que dichos patrones están relacionados con los gradientes ambientales más importantes.

\section{Material y métodos}

LAS ESPECIES Y EL ÁREA DE ESTUDIO

El objeto de estudio de este trabajo es la distribución de las 70 especies de micromamíferos en

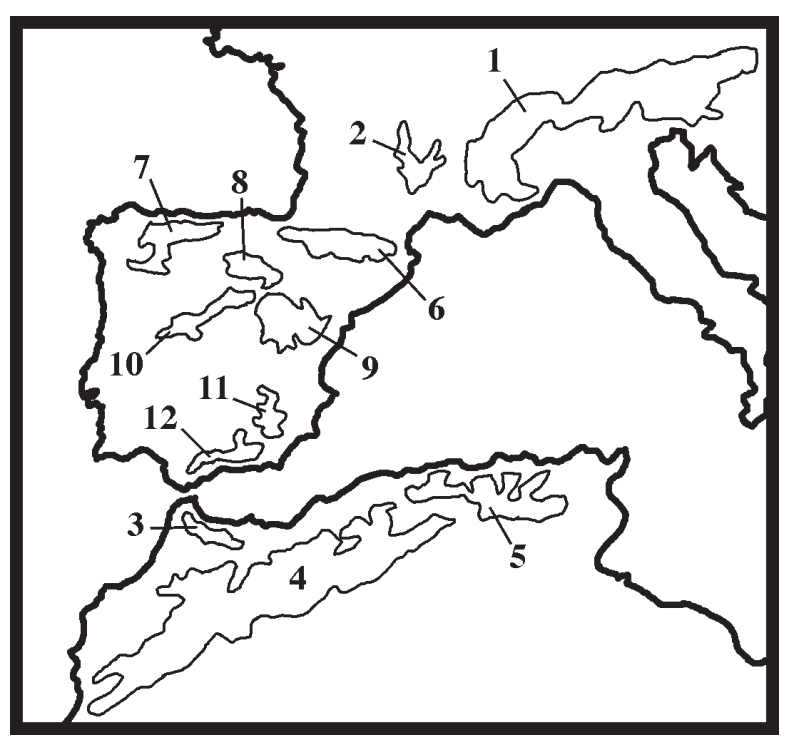

Fig. 1.- Sistemas montañosos considerados en el estudio. 1: Alpes; 2: Macizo Central Francés; 3: Rif; 4: Gran Atlas; 5: Atlas Telliano; 6: Pirineos; 7: Cantábrica; 8: S. Ibérico Norte; 9: S. Ibérico Sur; 10: Central; 11: C. Béticas; 12: C. Penibéticas.

Fig. 1.- Mountain ranges studied: 1: Alps; 2: French Masiff Central; 3: Riff; 4: High Atlas; 5: Tell Atlas; 6: Pyrenees; 7: Cantabrian Mts.; 8: Northern Iberian M.; 9: Southern Iberian M.; 10: Central Mts.; 11: Baetic Mts.; 12: Penibaetic Mts.

los sistemas montañosos que circundan la porción más occidental de la cuenca mediterránea. El término micromamíferos abarca tres órdenes distintos: Erinaceomorpha ( 7 especies en el área considerada), Soricomorpha (13 especies) y Rodentia (50 especies) (Apéndice 1). A efectos operativos, los sistemas montañosos considerados son las unidades territoriales discretas comprendidas entre la cota basal del piedemonte y el pico de máxima altitud en los Alpes, el Macizo Central Francés, los Pirineos, la Cordillera Cantábrica, el Sistema Ibérico Norte, el Sistema Ibérico Sur, el Sistema Central, las Sierras Béticas, las Sierras Penibéticas, el Rif, el Atlas Telliano y el Gran Atlas (incluyéndose en este último el Atlas Medio, el Atlas Sahariano y el Anti Atlas) (Fig. 1). En ellos se encuentran representadas todas las especies de micromamíferos del contexto geográfico estudiado, dado el carácter eurihípsico de la mayoría de los taxones que habitan en los medios montañosos mediterráneos (Pleguezuelos y Villafranca, 1997). Los datos relativos a la distribución de las especies proceden de las fuentes siguientes: Aulagnier y Thevenot (1986), Kowalski y Rzebik-Kowalska 
(1991), Mitchel-Jones et al. (1999) y Palomo y Gisbert (2002).

\section{LOS COROTIPOS}

El método utilizado para detectar los tipos de distribución, o corotipos, de micromamíferos en los sistemas montañosos se basa en un análisis de clasificación de las distribuciones de estas especies, siguiendo el procedimiento utilizado por Márquez et al. (1997) y Vargas et al. (1997). A continuación se describe dicha metodología de forma sucinta, haciendo énfasis tan sólo en aquellos aspectos de la misma que son necesarios para la comprensión de los resultados expuestos.

El primer paso ha consistido en la construcción de una matriz de similitudes entre las distribuciones de las 70 especies de micromamíferos en los sistemas montañosos. Para ello se ha utilizado el coeficiente de Baroni-Urbani y Buser (1976).

$$
I B=\frac{\sqrt{\left(C^{*} D\right)}+C}{\sqrt{\left(C^{*} D\right)}+A+B-C}
$$

donde $\mathrm{A}$ es el número de sistemas montañosos ocupados por la especie a, B es el número de sistemas montañosos con presencia de la especie b, C el número de montañas ocupados simultáneamente por las especies a y $\mathrm{b}$, mientras que $\mathrm{D}$ es el número de sistemas montañosos donde no aparecen ni la especie a ni la $b$.

A partir de la matriz de similitudes se han definido, a su vez: (1) un dendrograma de clasificación de las distribuciones, mediante la aplicación del algoritmo UPGMA (del inglés Unweighted Pair-Group Method using Arithmetic Averages, Sneath y Sokal, 1973), que es el método de clasificación aglomerativo que provoca la menor distorsión con respecto a las semejanzas originales entre los datos; (2) una matriz de similitudes significativas entre dichas distribuciones, en la cual los valores del coeficiente de BaroniUrbani y Buser se han sustituido por "+" cuando han sido mayores, "." si han sido menores, ó "0" si han sido similares con respeto a lo esperado por azar (Márquez et al. 1997). La significación estadística del coeficiente de similitud se ha basado en la tabla de valores críticos de Baroni-Urbani and Buser (1976). A través de los grupos de distribuciones reunidos por el dendrograma de clasificación, se ha explorado la existencia de corotipos siguiendo el método utilizado por Márquez et al. (1997) y Vargas et al. (1997).

En la matriz de similitudes significativas, los signos "+", "-" y "0" que relacionan entre sí distri- buciones de un grupo A del dendrograma están contenidos en una submatriz AxA; del mismo modo, los signos que relacionan distribuciones de un grupo $\mathrm{B}$ se encuentran en una submatriz BxB, y los que relacionan distribuciones del grupo $\mathrm{A}$ con las del grupo B se encuentran en una submatriz AxB. Teniendo esto en cuenta, se define el parámetro DW(AxA) como el grado en que las similitudes mayores que lo esperado por azar $(+)$ se hallan en la zona AxA pero no en la zona AxB, al mismo tiempo que las similitudes menores que lo esperado por azar (-) están ausentes de AxA; $\mathrm{DW}(\mathrm{BxB})$ es análogo a DW(AxA), si bien referido a la zona $\mathrm{BxB}$. Ambos parámetros combinan las condiciones necesarias para que un grupo de distribuciones A o B sean consideradas un corotipo, respectivamente.

DW (AxA) y DW (BxB) han sido calculados para todos los nodos del dendrograma, de forma que se ha considerado que un grupo de distribuciones es candidato para formar un corotipo si su DW(AxA) -o DW(BxB)- ha sido el mayor para todas las distribuciones involucradas en el grupo. Al mismo tiempo se ha definido, para cada grupo del dendrograma, el estadístico GW(AxA) -o GW(BxB), aplicando la prueba de independencia de la G (Sokal y Rohlf, 1981; McCoy et al., 1986) a la distribución de los signos "+" y "-" en las tres zonas AxA, BxB y AxB. Finalmente, un grupo A se ha considerado corotipo si se cumple al menos una de las condiciones siguientes: (1) su DW(AxA) es igual 0.7071 (máximo valor de DW posible); (2) su DW(AxA) es mayor que 0 y su correspondiente estadístico GW(AxA) es significativo. Las distribuciones que incumplen las condiciones descritas se han considerado "no agrupadas", de forma que no han constituido corotipo.

Se han calculado también los parámetros $\mathrm{DS}(\mathrm{AxA})$ y $\mathrm{DS}(\mathrm{BxB})$, que miden en qué grado las similitudes menores que lo esperado por azar (-) están en la zona $\mathrm{AxB}$, pero no en $\mathrm{AxA}$ o $\mathrm{BxB}$, y las similitudes mayores que lo esperado por azar $(+)$ están ausentes de la zona AxB. Estos parámetros combinan las condiciones necesarias para considerar que un corotipo presenta una segregación fuerte con respecto a las otras distribuciones de su nodo. El contraste estadístico de estos parámetros se ha realizado también mediante la prueba de la $\mathrm{G}$ (parámetro GS).

\section{CARACTERIZACIÓN AMBIENTAL DE LOS COROTIPOS}

Utilizando los métodos de regresión logística y análisis discriminante, se han buscado los modelos matemáticos que mejor definan las dife- 
rencias entre los sistemas montañosos incluidos en cada corotipo y aquellos que no lo están, en función de determinadas variables ambientales. Dichas variables han sido las siguientes: temperatura media anual $(\mathrm{T})$, temperatura media de julio (TJ), temperatura media de enero (TE) y evapotranspiración potencial media (ETP), para poner a prueba la importancia de la disponibilidad de energía en la configuración de los patrones biogeográficos; precipitación media anual $(\mathrm{P})$ para estimar la importancia de la disponibilidad de agua; evapotranspiración real media (ETR) para poner a prueba la importancia de la disponibilidad simultánea de agua y energía; y rango anual de temperatura (RT) para probar la importancia de la estabilidad climática.

Tanto en las regresiones logísticas como en los análisis discriminantes, la significación estadística del modelo se ha contrastado mediante la prueba de $\chi^{2}$. La incorporación de variables en los modelos se ha llevado a cabo por pasos, utilizando, en el caso de la regresión logística, el criterio de máxima verosimilitud (maximum likelihood criterion), contrastado, a su vez, mediante el estadístico de la relación de verosimilitudes (LR, del inglés loglikelihood-ratio). En el caso del análisis discriminante se ha empleado el estadístico lambda de Wilks.

Tanto en las regresiones logísticas como en los análisis discriminantes, se ha tenido en cuenta la posibilidad de que los corotipos estén relacionados de forma unimodal con las variables ambientales descritas. Para ello, se ha analizado cada corotipo en relación con cada variable ambiental por separado, considerando también el valor de ésta al cuadrado. En caso de obtenerse modelos significativos basados en la combinación cuadrática unimodal con alguna variable, dicha combinación se ha incluido, como una variable más, en el procedimiento por pasos que conduce al modelo de caracterización final.

Sólo se ha optado por utilizar los modelos discriminantes en ausencia de una función logística significativa.

\section{CARACTERIZACIÓN AMBIENTAL DE LA RIQUEZA DE ESPECIES}

Se ha explorado la relación de la riqueza de especies de micromamíferos con los principales gradientes ambientales, utilizando regresiones lineales y polinomiales de segundo y de tercer grado. Las variables consideradas son las descritas en el apartado anterior, a las que se han añadido: la altitud máxima de los sistemas montañosos
(AM), como estimador de la heterogeneidad ambiental; el área de los sistemas (A), como estimador de los factores implicados en la colonización histórica de las "islas" de territorio consideradas (MacArthur y Wilson, 1967); y las distancias mínimas a los sistemas montañosos situados a ambos extremos del área de estudio, es decir, distancia mínima a los Alpes (DA), distancia mínima al Gran Atlas (DGA), y distancia mínima a cualquiera de los dos (DAGA).

Se han analizado todas las especies de micromamíferos conjuntamente, y también el número de especies de roedores y de no roedores por separado. La decisión sobre qué modelo de regresión es el mejor, en caso de haber varios significativos, se ha tomado en función del parámetro $\mathrm{F}_{\mathrm{s}}$. Se ha desestimado el uso de la $\mathrm{R}^{2}$ por estar involucrados en la comparación modelos con diferentes grados de libertad.

\section{Resultados}

\section{LOS COROTIPOS}

A través de la clasificación de las distribuciones de los micromamíferos sobre las montañas del Paleártico Occidental, es posible distinguir cinco corotipos que incluyen la distribución de 49 especies, mientras que las 21 distribuciones restantes permanecen sin agrupar (ver la Tabla 1, la Figura 2, y el Apéndice 2). En términos biogeográficos se puede hablar de la existencia de un corotipo ampliamente distribuido (I), dos corotipos ibéricos (II yIII), un corotipo alpino (IV), y un corotipo africano $(\mathrm{V})$.

Tabla 1.- Valores de DW(AxA) en cada rama del dendrograma de la figura 1 , y valores de DS(AxA) para cada corotipo. M: máximo valor posible; ${ }^{* *}: \mathrm{p}<0.01 ; .^{* *}: \mathrm{p}<0.001$; N.S.: $\mathrm{p}>0.05$.

Table 1.- DW(AxA) values in each branch of the dendrogram in figure 1, and DS(AxA) values for each chorotype. M: maximum value possible; **: $\mathrm{p}<0.01 ;$.***: $\mathrm{p}<0.001 ;$ N.S.: $\mathrm{p}>0.05$.

\begin{tabular}{lcccc}
\hline & $\begin{array}{c}\text { Segregación débil } \\
\text { DW(AxA) }\end{array}$ & \multicolumn{2}{c}{$\begin{array}{c}\text { Segregación fuerte } \\
\text { DS(AxA) }\end{array}$} \\
\hline Corotipo I & 0,626 & $* * *$ & 0,000 & N.S. \\
Corotipo II & 0,707 & M & 0,000 & N.S. \\
Corotipo III & 0,707 & M & 0,000 & N.S. \\
Corotipo IV & 0,534 & $* * *$ & $-0,126$ & $* *$ \\
Corotipo V & 0,624 & $* * *$ & 0,686 & $* * *$ \\
\hline
\end{tabular}




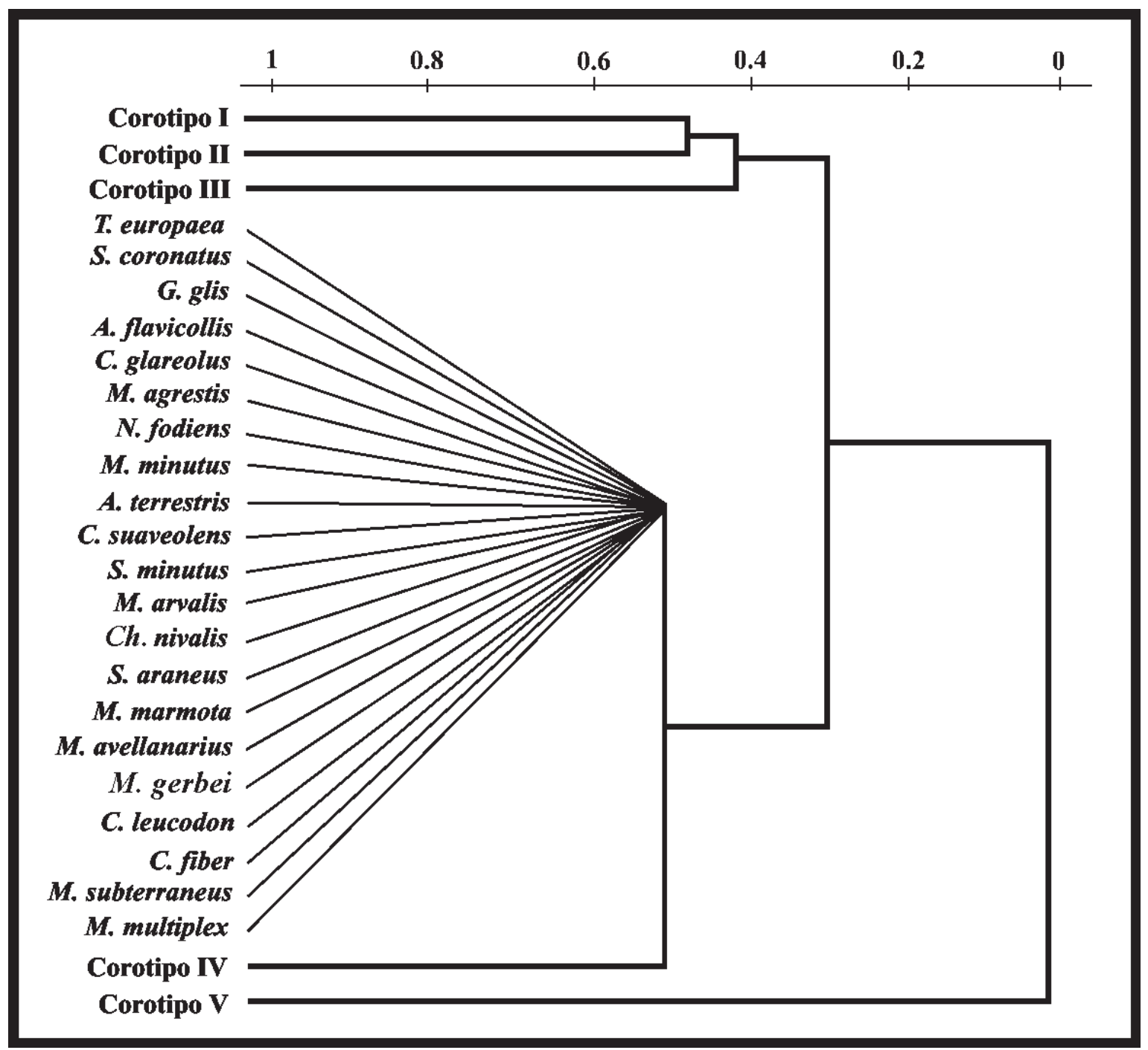

Fig. 2.- Dendrograma de clasificación de las distribuciones de micromamíferos en los sistemas montañosos.

Fig. 2.- Classification dendrogram of the micromammal distributions on the mountain ranges.

El corotipo I está formado por 14 especies presentes en casi todos los sistemas montañosos del área de estudio, si bien seis de ellas no se encuentran en las montañas africanas. Su caracterización ambiental no es posible debido a la ausencia de sistemas en los que falta dicho corotipo. Los corotipos II y III son los únicos propiamente ibéricos: el corotipo II está presente en los cuatro sistemas montañosos ibéricos más septentrionales, precisamente aquéllos que presentan una disponibilidad intermedia de agua procedente de la lluvia (P) dentro del área de estudio (Tabla 2); el corotipo III refleja la distribución del roedor Microtus cabrerae Thomas, 1906, un relicto ibérico presente en los Pirineos, en los sistemas Central e Ibérico Sur y en las sierras Bética y Penibética, cuya distribución geográfica ha sido imposible caracterizar en términos ambientales con las variables utilizadas. El corotipo IV lo forman ocho especies distribuidas por la mitad oriental del continente europeo y presentes, además, en los Alpes. Este sistema montañoso presenta como característica ambiental diferenciadora una precipitación $(\mathrm{P})$ y una inestabilidad cli- 
Tabla 2.- Caracterización ambiental de los corotipos de micromamíferos. R.L.: regresión logística; A.D.: análisis discriminante; **: $\mathrm{p}<0.01$; T: temperatura media anual; RT: rango anual de temperatura; P: precipitación media anual; $\mathrm{P}^{\wedge}$ : función polinomial de segundo grado de $\mathrm{P}\left(\mathrm{y}=-31,20+0,075 \mathrm{P}-0,000041 \mathrm{P}^{2}\right)$.

Table 2.- Environmental characterisation of micromammal chorotypes. R.L.: logistic regression; A.D.: discriminant analysis; **: $\mathrm{p}<0.01$; T: mean annual temperature; RT: annual temperature range; $\mathrm{P}$ : mean annual precipitation; $\mathrm{P}^{\wedge}$ : second degree polinomial function of $\mathrm{P}\left(\mathrm{y}=-31,20+0,075 \mathrm{P}-0,000041 \mathrm{P}^{2}\right)$.

\begin{tabular}{ccccc}
\hline Corotipo & Modelo & Tipo de modelo & $\chi^{2}$ & Signif. \\
\hline I & & & & $*$ \\
II & $y=0,1980+1,0123 \mathrm{P}^{\wedge}$ & R.L. & 11,457 & $* *$ \\
III & & & 14,437 & $* *$ \\
IV & $y=-7,608+0,0045 \mathrm{P}+0,233 \mathrm{RT}$ & A.D. & 8,268 & $* *$ \\
V & $\mathrm{y}=-12,652+0,899 \mathrm{~T}$ & R.L. & \\
\hline
\end{tabular}

mática (RT) mayor (Tabla 2) que el resto del área de estudio. Por último, las 23 distribuciones que configuran el corotipo $\mathrm{V}$ se restringen a las montañas africanas -16 especies son exclusivas del Gran Atlas-, ocupando así los sistemas montañosos del Paleártico occidental con mayor disponibilidad de energía (T) (Tabla 2) (Fig. 3).

Las 21 especies no agrupadas en corotipos presentan un patrón de distribución aparentemente continuo, ya que todas ellas están presentes en los sistemas europeos no ibéricos, y su número va disminuyendo gradualmente hacia las áreas más meridionales: 17 en los Pirineos, 13 en la Cordillera Cantábrica, nueve en el Sistema Ibérico Norte, cuatro en el Sistema Central, y una en el Sistema Ibérico Sur y en las Sierras Penibéticas. No se ha constatado la presencia de ninguna de estas especies en los sistemas montañosos norteafricanos.

\section{LA RIQUEZA DE ESPECIES}

En cualquiera de los tres casos analizados (micromamíferos, roedores y no roedores), el modelo de regresión más significativo (con una $\mathrm{F}_{\mathrm{s}}$ mayor) sobre las variables ambientales y geográficas consideradas ha sido una curva polinomial de tercer grado (Fig. 4). Para los micromamíferos no roedores y para el conjunto total de especies la mejor regresión se establece en relación con un gradiente de disponibilidad de energía (temperatura media de julio, TJ, con un $\mathrm{r}^{2}$ mayor que 0,90 ). En ambos casos, TJ propicia un ligero incremento del número de especies desde los Pirineos hasta los Alpes. A partir de aquí se registra un decrecimiento de la riqueza específica conforme aumenta $\mathrm{TJ}$, siguiendo claramente una tendencia latitudinal hacia el sur. Al llegar a los sistemas africanos más meridionales, no obstante, las curvas experimentan un nuevo crecimiento hacia el Gran Atlas. Procede destacar que este macizo montañoso, a pesar de presentar una TJ mayor que el resto, alberga una importante riqueza específica de micromamíferos.

Para los roedores, la disponibilidad simultánea de agua y energía $\left(E T R, \mathrm{r}^{2}=0,91\right)$ es el factor que mejor explica la variación de la riqueza de especies, si bien seguido muy de cerca por la temperatura media de julio $\left(r^{2}=0,86\right)$ (Fig. 4). La proyección de los diferentes sistemas montañosos sobre la curva es semejante a la descrita en el párrafo anterior, si bien la tendencia latitudinal de la riqueza de especies está vinculada, en este caso, al decrecimiento de ETR hacia el sur.

\section{Discusión}

Vargas et al. (2003) plantean la hipotética existencia de dos núcleos principales de sinapocorías para los micromamíferos, uno entre los sistemas montañosos de la Europa extrapeninsular y otro entre los sistema montañosos del norte de Affrica, para explicar el patrón de regionalización de los sistemas montañosos de la Península Ibérica y su entorno. Esta interpretación se contrapone a la existencia de un importante centro de endemismos ibéricos para la fauna de micromamíferos, como se ha puesto de manifiesto para los anfibios (Real et al., 1992). Los resultados aquí obtenidos corroboran la primera hipótesis, tanto en lo que respecta al análisis de los corotipos como al de la distribución de la riqueza de especies. 


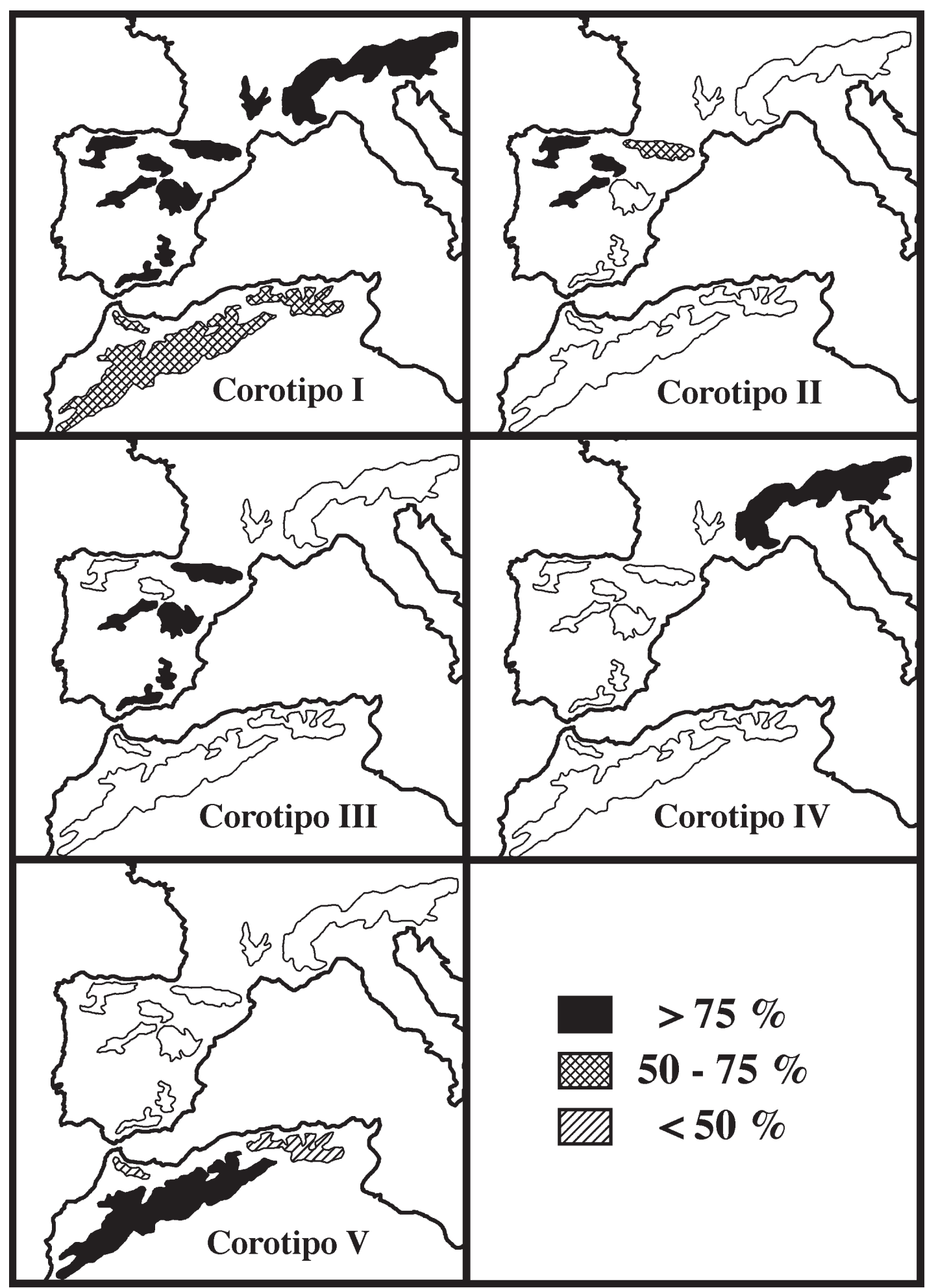

Fig. 3.- Distribución geográfica de los corotipos de micromamíferos en los sistemas montañosos.

Fig. 3.- Geographic distribution of the micromammal chorotypes in the mountain ranges. 


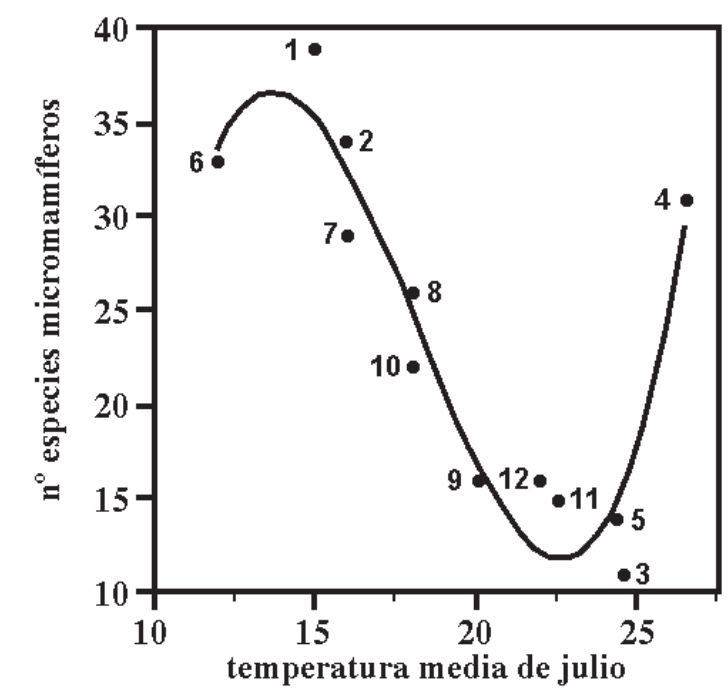

$\mathrm{SM}=-315,95+64,38 \mathrm{~T} . \mathrm{J}-3,77 \mathrm{~T} . \mathrm{J}^{2}+0,069 \mathrm{~T} . \mathrm{J}^{3}$

$$
\mathbf{r}^{2}=0,91
$$

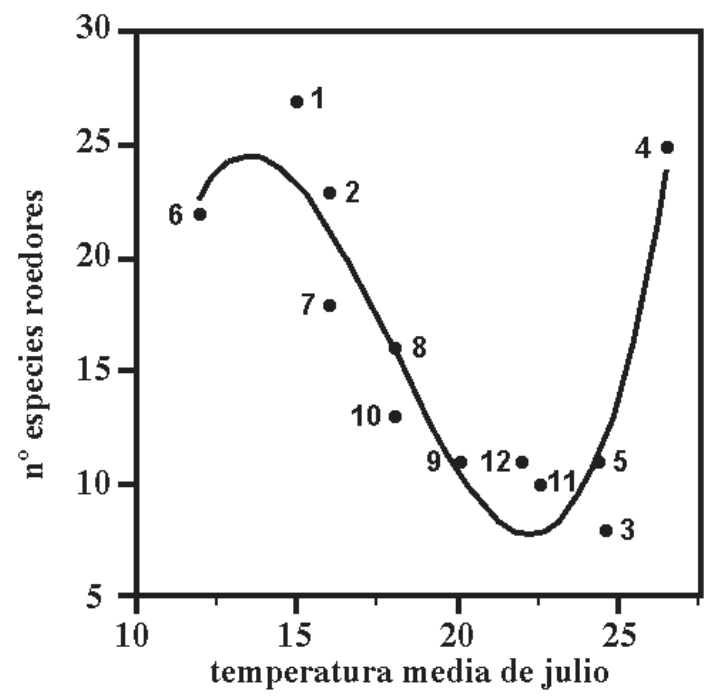

$\mathrm{SR}=-227,06+46,42 \mathrm{TJ}-2,75 \mathrm{TJ}^{2}+0,051 \mathrm{TJ}^{3}$

$$
\mathbf{r}^{2}=0,86
$$

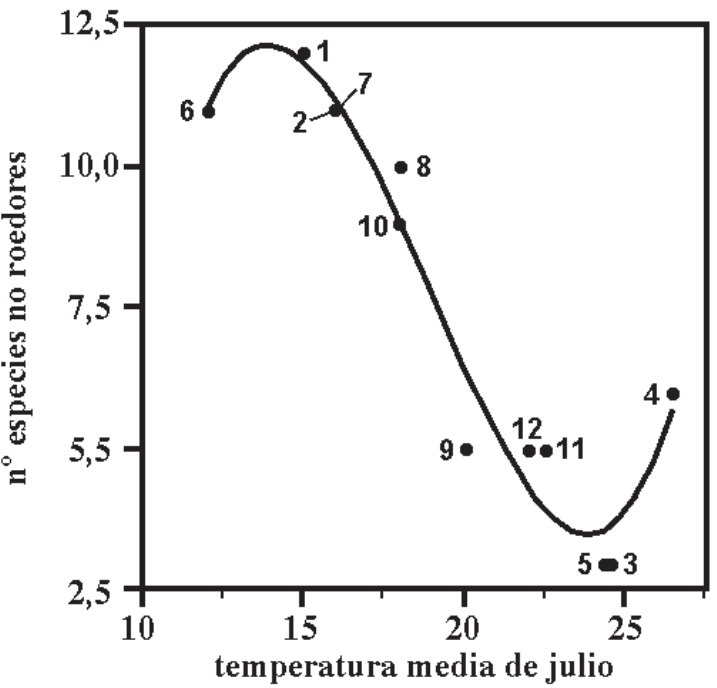

SN=-88,89+17,96T.J-1,02 T.J ${ }^{2}+0,018 \mathrm{~T}^{3} \mathrm{~J}^{3}$

$$
\mathbf{r}^{2}=0,96
$$

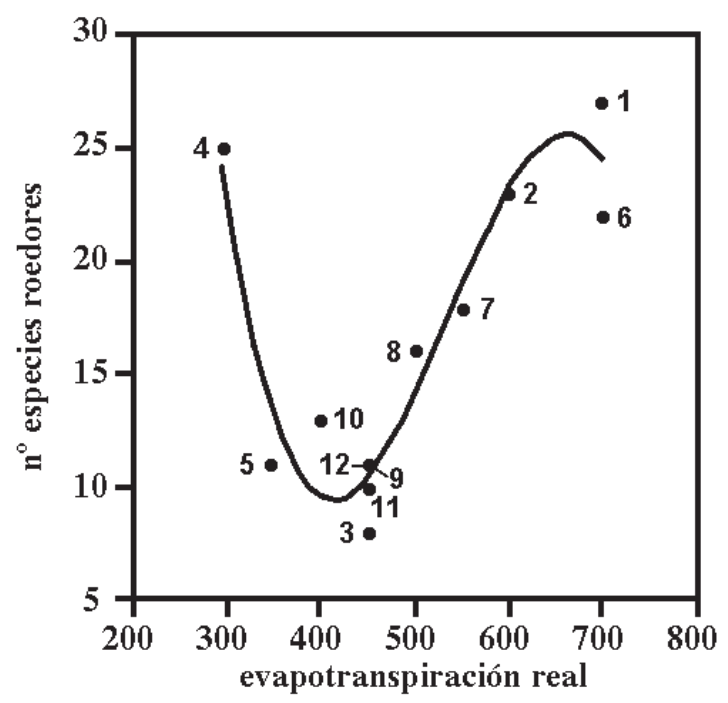

$\mathrm{SR}=291,13-1,72 \mathrm{ETR}+0,0034 \mathrm{ETR}^{2}$ $-0,000002 \mathrm{ETR}^{3}$

$$
\mathbf{r}^{2}=0,91
$$

Fig. 4.- Ajuste polinomial de tercer grado de la riqueza de micromamíferos (SM), de roedores (SR) y de micromamíferos no roedores $(\mathrm{SN})$ sobre la temperatura media de julio (TJ), y de los roedores sobre la evapotranspiración real media (ETR).

Fig. 4. - Third degree polinomial fit of the species richness of micromammals (SM), rodents (SR) and non rodent micromammals (SN) on the mean July Temperature (TJ), and of rodents on the mean actual evapotranspiration (ETR). 


\section{LOS COROTIPOS}

El corotipo I incluye 14 especies de amplia distribución por los sistemas montañosos analizados, de forma que aporta poca información sobre posibles rutas de dispersión o tendencias de retracción de las distribuciones. Estas especies, que penetraron desde Europa, desde África o desde la Península Ibérica hacia África y el resto de Europa, han alcanzado un mismo tipo de distribución cosmopolita, o casi cosmopolita, en el contexto geográfico considerado. En todo caso, este corotipo cosmopolita sí pone de manifiesto la existencia de intercambios de fauna entre todos los sistemas montañosos más occidentales de la cuenca mediterránea.

Únicamente dos corotipos, el II y el III, son exclusivamente ibéricos y, entre ambos, solo reúnen cuatro de las 70 especies analizadas, lo que sugiere que la Península Ibérica no alberga una biota endémica importante de micromamíferos.

El corotipo IV está formado por 8 especies alpinas que no se han dispersado hacia el resto de sistemas montañosos del área de estudio, por lo que no aportan información sobre rutas de dispersión, aunque sí manifiesta la importancia que tienen los Alpes para los micromamíferos. De forma análoga, las 23 especies del corotipo $\mathrm{V}$ ponen de manifiesto la importancia del Gran Atlas para los micromamíferos, ya que todas ellas están presentes en este macizo montañoso, aunque sólo siete alcanzan las otras montañas africanas y ninguna llega a cruzar el Estrecho. De esta forma, los corotipos IV y V destacan la mayor importancia de los polos centroeuropeo y africano respecto al papel específico de la fauna ibérica representada por los corotipos II y III.

Las 21 especies que no han podido ser agrupadas en ningún corotipo responden a un patrón de distribución continuo o gradual. Este tipo de coexistencia entre corotipos y reemplazamientos graduales de distribuciones ya fue encontrado por Real et al. (1997) para los reptiles del Rif marroquí. Estos autores interpretaron las distribuciones de reptiles que siguen un patrón gradual como carentes de equilibrio biogeográfico, debido a que diversas especies se encontraban en proceso de penetración desde el Sahara hasta el Rif. Esta misma interpretación puede ser invocada para explicar el patrón gradual de las 21 especies de micromamíferos en el área de estudio aquí considerada. Dado que todas ellas se hallan en los Alpes y que, por tanto, están directamente relacionadas con el corotipo IV (ver Fig. 2), probablemente constituyen un conjunto de especies que se encuentra en proceso de dispersión desde los Alpes hacia la Península Ibérica, o bien de retracción desde la Península hacia los Alpes. Al menos para una de estas especies, Crocidura suaveolens (Pallas, 1811), hay evidencia de que su área de distribución en la Península Ibérica era más amplia durante el Pleistoceno Superior que en la actualidad (Ruiz Bustos et al., 1984), de forma que su distribución se está restringiendo hacia el norte. Este fenómeno de retracción de las distribuciones a latitudes más septentrionales ha sido puesto también de manifiesto para alguna especies de aves (Sánchez, 1996).

\section{LA RIQUEZA DE ESPECIES}

El patrón de riqueza específica en el área de estudio presenta una tendencia bimodal, ya que el número de especies disminuye gradualmente de norte a sur pero se incrementa de nuevo en el Gran Atlas. En el ámbito europeo, a los sistemas no ibéricos (39 especies en los Alpes y 34 en el Macizo Central Francés) le siguen, en orden decreciente de riqueza, los Pirineos, la Cordillera Cantábrica, el Sistema Ibérico Norte y el Sistema Central. Esto concuerda con el patrón encontrado por Krystufek y Griffiths (2002) para los roedores en Europa, en el que el punto más occidental de alta diversidad de especies se encuentra en los Alpes. Los sistemas más meridionales, es decir, El Rif y el Atlas Telliano presentan la menor riqueza (11 y 14 especies, respectivamente). Sin embargo, el Gran Atlas rompe bruscamente este patrón latitudinal al situarse, con 31 especies de micromamíferos, por encima de todos los sistemas exclusivamente ibéricos. El patrón bimodal se mantiene tanto para el orden Rodentia como para los no roedores. En ambos casos, si bien de forma menos drástica en el caso de los no roedores, el Gran Atlas rompe con la tendencia geográfica a disminuir el número de especies en sentido norte-sur. Este patrón bimodal, con los máximos en los puntos extremos, podría evidenciar el papel de los sistemas montañosos del resto de Europa y de África como pasos en la ruta de dispersión de especies hacia la Península Ibérica, o como zonas de acumulación de especies que, en otro tiempo, tuvieron una distribución ibérica más amplia.

Queda por dilucidar si los dos núcleos de intercambio se pueden relacionar también con dos biotas de orígenes diferentes para los micromamíferos. La relación del número de especies con las variables ambientales puede arrojar luz sobre esta cuestión. La hipótesis de la favorabilidad-adversidad ambiental de Richerson y Lum 
(1980) mantiene que cuando los parámetros ambientales se acercan a los valores óptimos para los requerimientos fisiológicos de un grupo de especies, los organismos se pueden especializar respecto a más gradientes físicos y dedicar mayor cantidad de energía, materia y genoma a los ajustes coadaptativos con otras especies, por lo que la riqueza específica aumenta. Richerson y Lum (1980) interpretan los valores medios de las variables ambientales como índices adecuados para poner a prueba la hipótesis de la favorabilidad. Terborgh (1973) mantenía que los conceptos de favorabilidad y de adversidad reflejan las tasas relativas de especiación o colonización y de extinción en diferentes hábitats o regiones geográficas. Según este enfoque, ambientes adversos reducen las tasas de especiación o colonización e incrementan las tasas de extinción. Un origen diferente de la fauna de micromamíferos, en lugares latitudinalmente distantes, implicaría que sus óptimos fisiológicos, y las tasas de colonización y extinción relacionadas con ellos, se situarían en puntos separados del gradiente definido por algunas variables ambientales. De acuerdo con este planteamiento, un origen dual de la fauna que la Península intercambia con el norte y el sur debería poder relacionarse con un comportamiento bimodal del número de especies respecto a algún factor ambiental, y no sólo con las distancias y las barreras geográficas.

La Figura 3 muestra que el comportamiento geográfico bimodal del número de especies se corresponde con una respuesta bimodal significativa respecto a la temperatura estival y la evapotranspiración real. Esto sugiere que los dos núcleos principales de intercambio faunístico se corresponden con dos biotas diferentes, de forma que la fauna de origen europeo presenta su óptimo ambiental en valores de temperatura media de julio en torno a los $15^{\circ} \mathrm{C}$ mientras que la fauna de origen africano lo presenta por encima de $\operatorname{los} 25^{\circ} \mathrm{C}$.

\section{AGRADECIMIENTOS}

Agradecemos al Dr. Juan J. Morrone la revisión crítica que realizó de una primera versión del manuscrito.

\section{Referencias}

Aulagnier, S. \& Thevenot, M., 1986. Catalogue des mamiferes sauvages du Maroc. Travaux de L'Institut Scientifique, Série Zoology No 41. Rabat. 142 pp.
Baroni-Urbani, C. \& Buser, M. W., 1976. Similarity of binary data. Systematic Zoology, 25: 251-259.

De Lattin, G., 1967. Grundriss der Zoogeogeographie. Veb Gustav Fischer Verlag. Jena. 602 pp.

KowAlski, K. \& RZEBIK-KowAlsKa, B., 1991. Mammals of Algeria. Ossolineum. Wroclaw. 370 pp.

KrystufEK, B. \& GRIFFITHS, H. I., 2002. Species richness and rarity in European rodents. Ecography, 25: 120-128.

MacArthur, R. R. \& Wilson, E. O., 1967. The theory of Island Biogeography. Princenton University Press. Princenton. 203 pp.

Márquez, A. L., Real, R., Vargas, J. M. \& Salvo, A. E., 1997. On identifying common distribution patterns and their causal factors: a probabilistic method applied to pteridophytes in the Iberian Peninsula. Journal of Biogeography, 24: 613-631.

McCoy, E. D., Bell, S. S. \& Walters, K., 1986. Identifying biotic boundaries along environmental gradients. Ecology, 67: 749-759.

Mitchel-Jones, A. J., Amori, G., Bogdanowicz, W., Krustufek, B., Reijnders, P. J. H., Spitzenberger, F., Stubbe, M., Thissen, J. B. M., Vohralik, V. \& ZimA, J., 1999. Atlas of the European Mammals. Academic Press. London. 496 pp.

Palomo, L. J. \& Gisbert, J. (eds.), 2002. Atlas de los Mamiferos terrestres de España. Dirección General de Conservación de la Naturaleza-SECEM-SECEMU. Madrid. 564 pp.

Pleguezuelos, J. M. \& Villafranca, C., 1997. Distribución altitudinal de la herpetofauna ibérica. In: J. P. Martínez-Rica \& J. M. Pleguezuelos (eds.). Distribución y Biogeografía de los Anfibios y Reptiles de España y Portugal. Universidad de Granada y Asociación Herpetológica Española. Granada: 321-341.

Real, R., Antúnez, A. \& Vargas, J. M., 1992. A biogeographic synthesis of European amphibians. In: Z. Korsós \& I. Kiss (eds.). Proceedings of the $6^{\text {th }}$. Ordinary General Meeting of the Societas Europaea Herpetologica. 19-23 August 1991, Budapest. Hungarian Natural History Museum. Budapest: 377-381.

Real, R., Pleguezuelos, J. M. \& Fahd, S., 1997. The distribution patterns of reptiles in the Riff region, northern Morocco. African Journal of Ecology, 35: 312-325.

Richerson, P. J. \& LuM, K., 1980. Patterns of plant species diversity in California: relation to weather and topography. American Naturalist, 116: 504-536.

Ruiz-Bustos, A., Vargas, J. M., Camprodón, J. \& SansComa, V., 1984. Die Gartenspitzmaus, Crocidura suaveolens (Pallas, 1811) im Jungpleistozän (Würm I) von Südspanien. Säugetierkundliche Mitteilungen, 31: 251-256. 
SÁNCHEZ, A., 1996. Aves fósiles del Pleistoceno ibérico: rasgos climáticos, ecológicos y zoogeográficos. Ardeola, 43: 207-219.

SNeATH, P. H. A. \& SoKal, R. R., 1973. Numerical taxonomy. The principles and practice of numerical classification. W. H. Freeman. San Francisco. 573 pp.

SoKal, R. R. \& RoHLF, F. J., 1981. Biometry: The principles and practice of statistics in biological research. W. H. Freeman \& Company. New York. 859 pp.

Terborgh, J., 1973. On the notion of favorableness in plant ecology. American Naturalist, 107: 481-501.
Vargas, J. M., Olivero, J., Márquez, A. L., Guerrero, J. C. \& REAL, R., 2003. Relaciones biogeográficas entre los sistemas montañosos de la Península Ibérica y su entorno: el caso de los micromamíferos. Graellsia, 59(2-3): 319-329.

Vargas, J. M., Real, R. \& Palomo, L. J., 1997. On identifying significant co-occurrence of species in space and time. Miscel-lània Zoològica, 20(2): 49-58.

Apendice 1.- Lista de especies consideradas en el estudio.

Appendix 1.- Lists of species analysed.

Erinaceus europaeus Linnaeus, 1758

Atelerix algirus (Lereboullet, 1842)

Hemiechinus aethiopicus (Ehrenberg, 1832)

Galemys pyrenaicus (E. Geoffroy Saint-Hilaire, 1811)

Talpa europaea Linnaeus, 1758

Talpa occidentalis Cabrera, 1907

Talpa caeca Savi, 1822

Sorex minutus Linnaeus, 1766

Sorex araneus Linnaeus, 1758

Sorex coronatus Millet, 1828

Sorex granarius Miller, 1910

Sorex alpinus Schinz, 1837

Neomys anomalus, Cabrera, 1907

Neomys fodiens, (Pennant, 1771)

Suncus etruscus (Savi, 1822)

Crocidura suaveolens (Pallas, 1811)

Crocidura russula (Hermann, 1780)

Crocidura leucodon (Hermann, 1780)

Crocidura whitakeri de Winton, 1898

Crocidura lusitania Dollman, 1915

Sciurus vulgaris Linnaeus, 1758

Marmota marmota (Linnaeus, 1758)

Atlantoxerus getulus (Linnaeus, 1758)

Gerbillus campestris (Loche, 1867)

Gerbillus nanus Blanford, 1875

Gerbillus gerbillus (Olivier, 1801)

Gerbillus pyramidum Geoffroy, 1825

Gerbillus simoni Lataste, 1881

Pachyuromys duprasi Lataste, 1880

Meriones shawi (Duvernoy, 1842)

Meriones libycus Lichtenstein, 1823

Meriones crassus Sundevall, 1842

Psammomys obesus Cretzschmar, 1828

Castor fiber Linnaeus, 1758

Eliomys quercinus (Linnaeus, 1766)
Dryomys nitedula (Pallas, 1778)

Glis glis Linnaeus, 1766

Muscardinus avellanarius (Linnaeus, 1758)

Micromys minutus (Pallas, 1771)

Apodemus flavicollis (Melchior, 1834)

Apodemus sylvaticus (Linnaeus, 1758)

Apodemus alpicola Heinrich, 1952

Lemniscomys barbarus (Linnaeus, 1766)

Rattus rattus (Linnaeus, 1758)

Rattus norvegicus (Berkenhout, 1769)

Mus spretus Lataste, 1883

Mus musculus Linnaeus, 1758

Mus domesticus Schwarz \& Schwarz, 1943

Mastomys erythroleucus (Temminck, 1853)

Acomys cahirinus (Desmarest, 1819)

Jaculus jaculus Linnaeus, 1758

Jaculus orientalis Erxleben, 1777

Hystrix cristata Linnaeus, 1758

Ctenodactylus gundi (Rothmann, 1976)

Ctenodactylus vali (Thomas, 1902)

Clethrionomys glareolus (Schreber, 1780)

Arvicola sapidus Miller, 1908

Arvicola terrestris (Linnaeus, 1758)

Chionomys nivalis (Martins, 1842)

Microtus arvalis (Pallas, 1778)

Microtus cabrerae (Thomas, 1906)

Microtus agrestis (Linnaeus, 1761)

Microtus subterraneus (de Sélys-Longchamps, 1836)

Microtus multiplex (Fatio, 1905)

Microtus gerbei (Gerbe, 1879)

Microtus bavaricus Köning, 1962

Microtus duodecimcostatus de Sélys-Longchamps, 1839

Microtus lusitanicus (Gerbe, 1879)

Microtus savii (de Sélys-Longchamps, 1838)

Sicista betulina (Pallas, 1779) 
Apéndice 2.- Especies que forman cada uno de los cinco corotipos encontrados en los micromamíferos.

Appendix 2.- Species included in each chorotype.

\section{Corotipo I}

Erinaceus europaeus Linnaeus, 1758

Talpa occidentalis Cabrera, 1907

Neomys anomalus Cabrera, 1907

Suncus etruscus (Savi, 1822)

Crocidura russula (Hermann, 1780)

Sciurus vulgaris Linnaeus, 1758

Eliomys quercinus (Linnaeus, 1766)

Apodemus sylvaticus (Linnaeus, 1758)

Rattus rattus (Linnaeus, 1758)

Rattus norvegicus (Berkenhout, 1769)

Mus spretus Lataste, 1883

Mus domesticus Schwarz \& Schwarz, 1943

Arvicola sapidus Miller, 1908

Microtus duodecimcostatus de Sélys-Longchamps, 1839

\section{Corotipo II}

Galemys pyrenaicus (E. Geoffroy Saint-Hilaire, 1811)

Sorex granarius Miller, 1910

Microtus lusitanicus (Gerbe, 1879)

\section{Corotipo III}

Microtus cabrerae (Thomas, 1906)

\section{Corotipo V}

Atelerix algirus (Lereboullet, 1842)

Hemiechinus aethiopicus (Ehrenberg, 1832)

Crocidura whitakeri de Winton, 1898

Crocidura lusitania Dollman, 1915

Atlantoxerus getulus (Linnaeus, 1758)

Gerbillus campestris (Loche,1867)

Gerbillus nanus Blanford, 1875

Gerbillus gerbillus (Olivier, 1801)

Gerbillus pyramidum Geoffroy, 1825

Gerbillus simoni Lataste, 1881

Pachyuromys duprasi Lataste, 1880

Meriones shawi (Duvernoy, 1842)

Meriones libycus Lichtenstein, 1823

Meriones crassus Sundevall, 1842

Psammomys obesus Cretzschmar, 1828

Lemniscomys barbarus (Linnaeus, 1766)

Mastomys erythroleucus (Temminck, 1853)

Acomys cahirinus (Desmarest, 1819)

Jaculus jaculus Linnaeus, 1758

Jaculus orientalis Erxleben, 1777

Hystrix cristata Linnaeus, 1758

Ctenodactylus gundi (Rothmann, 1976)

Ctenodactylus vali (Thomas, 1902)

\section{Corotipo IV}

Sorex alpinus Schinz, 1837

Dryomys nitedula (Pallas, 1778)

Apodemus alpicola Heinrich, 1952

Mus musculus Linnaeus, 1758

Microtus bavaricus Köning, 1962

Microtus savii (de Sélys-Longchamps, 1838)

Sicista betulina (Pallas, 1779)

Talpa caeca Savi, 1822 